\title{
ON THE PRIMARY ORBITS OF STAR MAPS (FIRST PART)
}

Abstract. This paper is the first one of a series of two, in which we characterize a class of primary orbits of self maps of the 4-star with the branching point fixed. This class of orbits plays, for such maps, the same role as the directed primary orbits of self maps of the 3-star with the branching point fixed. Some of the primary orbits (namely, those having at most one coloured arrow) are characterized at once for the general case of $n$-star maps.

1. Introduction. The notion of a primary orbit has revealed to be very useful in the study of several characteristics of the dynamics of discrete onedimensional systems. This notion for interval maps is related to the notion of a minimal orbit (see, for instance, [12], [7], [8], [3], [9], [10], [11]). However it is more general since its definition depends only on the behaviour of a map from a given class on such an orbit. Hence it is possible to characterize these orbits by looking only at their shapes.

Primary orbits were introduced in [1] to study continuous self maps of the space consisting of three intervals joined by a common endpoint- the $\mathbb{Y}$ or the 3 -star - with this point fixed. This class of maps will be called $\mathcal{Y}$ in this paper. Simultaneously, Baldwin [5] considered the same notion for maps on the interval or the real line, which he called $\rightarrow$-minimal cycles. For interval maps, the primary (or minimal) orbits are shown by several authors to coincide with the simple ones (see [5] and [2]; see also [12], [7], [8], [3], [9], [10], [11]). For a definition of simple orbit see, for instance, [2]. These orbits were called pendulum in [1].

In this series of two papers we start the generalization of the characterization of primary orbits of maps from $\mathcal{Y}$ to self maps of the $n$-star

2000 Mathematics Subject Classification: Primary 37E15.

Key words and phrases: primary orbit, $n$-star, strongly directed orbit.

The authors have been partially supported by the DGES grant PB96-1153, the CONACIT grant 1999SGR-00349 and the CIRIT grant 2000SGR-00027. 
( $n$ intervals joined by a common endpoint) with the branching point fixed. In [1], the primary orbits were classified into two categories: directed and undirected. We are convinced that directed orbits are the "genuine" primary orbits in the $\mathbb{Y}$, whereas the undirected ones are "inherited" in some way from maps of the interval. However, when trying to do that generalization, one quickly sees that, for $n \geq 4$, directed primary orbits no longer have the good properties they had in $\mathbb{Y}$. These properties are based on the Directed Rule [1, Lemma 10.4], which the primary directed orbits of maps from $\mathcal{Y}$ satisfy. A crucial consequence of the Directed Rule is that the primary orbits of maps from $\mathcal{Y}$ cannot have more than two coloured arrows ([1, Directed Theorem (d)]). For $n \geq 4$ it is easy to find examples of directed primary orbits which do not satisfy those properties and, hence, the Directed Rule (see Example 4.13).

We generalize both the Directed Rule and the General Rule (Lemma 6.1) of [1] into a new condition we call the Generalized Directed Rule. The orbits satisfying this condition are called strongly directed. For such orbits, in the case of the 4-star, it is possible to show that they have at most three coloured arrows (Theorem 4.10). Therefore these orbits play the same role as the directed primary orbits of maps from $\mathcal{Y}$. This is the class of primary orbits we are going to study.

We feel that strongly directed orbits are the "genuine" primary orbits in the $n$-star. In particular we think that primary orbits which do not satisfy the Generalized Directed Rule (directed as well as undirected ones) arise, in some way, from stars with less branches. The understanding in depth of this point and the extension of the characterization of the primary orbits to orbits of the 4-star not satisfying the Generalized Directed Rule is an open problem.

The characterization of the primary orbits for $n \geq 4$ is essentially different from that for the case $n=3$. However, as will be apparent from Sections 4-6 and [4], the ideas and techniques used in the characterization of the strongly directed primary orbits for the case $n=4$ should allow us to find these orbits in the general case (which would be an essential contribution to the understanding of the primary orbits for the $n$-stars). In this spirit, this series of two papers should not be regarded as the sequel of the study of the primary orbits for the 3-star, neither as a natural (and perhaps boring) step to the study of the 5 -star, but rather as an attempt to understand in an easy case the ideas that should allow one to undertake the task in the general case. These papers are an essential step towards the characterization of the primary orbits of the $n$-stars.

To develop our study we classify the primary strongly directed orbits of the 4-star into several families, following the characterization of the directed primary orbits of maps from $\mathcal{Y}$. The first classes of primary orbits we find 
are twist orbits (Definition 3.1), having no coloured arrows, and single orbits (Definition 3.3), with only one coloured arrow. For these two simple types of orbits, we give a characterization which is independent of $n$. In Theorems 3.2 and 3.6 we show that they are the only primary orbits without coloured arrows or with just one coloured arrow, respectively.

For orbits with two or three coloured arrows the situation is a bit more complicated. To classify them we must look at another feature of its shape, namely whether they have or do not have crossing arrows. Theorem 5.8 characterizes the primary orbits having crossing arrows and some coloured arrow as box orbits (Definition 5.2).

Finally, the study of the primary strongly directed orbits without crossing arrows having more than one coloured arrow, requiring more specific techniques, is left for [4], the second paper of this series.

This paper is organized as follows. Section 2 gives the necessary definitions and general results for the study of strongly directed orbits. In Section 3 we study twist and single orbits. Section 4 is devoted to stating and proving the basic properties of strongly directed orbits, as well as to explaining some differences between these orbits and directed orbits not satisfying the Generalized Directed Rule. In Section 5 we study box orbits. Finally, in Section 6 we summarize the main results of this paper.

2. Definitions, notation and preliminary results. The symbols $\mathbb{N}$, $\mathbb{Z}$ and $\mathbb{Q}$ will denote, as usual, the sets of natural numbers (without zero), integers and rational numbers respectively. We often must deal with the ring of integers $\bmod k$ which we will denote by $\mathbb{Z}_{k}$. Then we use the symbols $\oplus$ and $\ominus$ (or $\oplus_{k}$ and $\ominus_{k}$, if necessary) to denote the addition and subtraction in $\mathbb{Z}_{k}$.

For $n \in \mathbb{N}$, the $n$-star is the subspace of the plane consisting of all complex numbers $z$ such that $z^{n}$ is in the unit interval $[0,1]$. We shall denote the $n$-star by $\mathbb{X}_{n}$. We shall also use the notation $\mathcal{X}_{n}$ to denote the class of all continuous maps from $\mathbb{X}_{n}$ to itself with 0 as a fixed point. In particular we have $\mathbb{Y}=\mathbb{X}_{3}$ and $\mathcal{Y}=\mathcal{X}_{3}$. We note that the 1 -star and the 2 -star are homeomorphic to a closed interval of the real line. Thus, in what follows, when talking about $\mathbb{X}_{n}$ or $\mathcal{X}_{n}$ we shall always assume that $n \geq 2$. The symbol $\equiv$ will denote congruence modulo $n$.

As usual, if $f \in \mathcal{X}_{n}$ we shall write $f^{k}$ to denote $f \circ \ldots \circ f$ ( $k$ times) when $k \in \mathbb{N}$, and $f^{0}=$ id. A point $x \in \mathbb{X}_{n}$ such that $f^{k}(x)=x$ for some $k \in \mathbb{N}$ will be called a periodic point of $f$. Then the set $\operatorname{Orb}_{f}(x)=\left\{f^{i}(x): i \in \mathbb{N}\right\}$ is a periodic orbit of $f$. The period of $x$ is $\operatorname{Card}\left(\operatorname{Orb}_{f}(x)\right)$ (usually we also call it the period of the orbit). Then $x$ has period $m$ if and only if $f^{m}(x)=x$ but $f^{j}(x) \neq x$ for $j=1, \ldots, m-1$. To simplify our study we will not consider $\{0\}$ as a periodic orbit of maps from $\mathcal{X}_{n}$. 
Although we study maps from $\mathcal{X}_{n}$, we need to consider simultaneously the family $\mathcal{I}$ of continuous functions of the interval $\mathbb{I}=[0,1] \subset \mathbb{R}$ into itself. Of course, for maps from $\mathcal{I}$ we use the same notation and terminology. If we do not need to be precise, $\mathbb{X}$ will represent $\mathbb{I}$ as well as $\mathbb{X}_{n}$, and $\mathcal{X}$ will represent $\mathcal{I}$ as well as $\mathcal{X}_{n}$.

Most of the basic definitions and results from [1] can be easily generalized to more complicated trees. In particular, [1, Sections 1, 2, 3 and 8] are immediately extended, mutatis mutandis, to the context of $n$-stars. Since this series of two papers is a continuation of [1], we shall freely use these results and definitions in the more general context of the $n$-stars without rewriting them. We only slightly modify (and recall) the notation from [1] as follows.

Following [1], $E$ will always denote the set $\{0\}$ (we only consider $n \geq 2$ ). The closures of components of $\mathbb{X} \backslash E$ are called branches. For $x \in \mathbb{X} \backslash E$ we denote by $\operatorname{br}(x)$ the branch where $x$ is, and we put $x \sim y$ if $\operatorname{br}(x)=\operatorname{br}(y)$.

Let $P$ be a subset of $\mathbb{X}$. The span of $P$ is the smallest closed connected subset of $\mathbb{X}$ containing $P$. It will be denoted by $\langle P\rangle$. When $P=\left\{x_{1}, \ldots, x_{k}\right\}$, we simply write $\left\langle x_{1}, \ldots, x_{k}\right\rangle$ for $\left\langle\left\{x_{1}, \ldots, x_{k}\right\}\right\rangle$.

An interval is a connected subset of a branch. Then the intervals in $\mathbb{X}_{n}$ are homeomorphic to intervals of the real line. Usually the word interval will mean closed interval, that is, a set of the form $\langle x, y\rangle$ where $x$ and $y$ are the endpoints of the interval and satisfy $x=0$ or $y=0$ or $x \sim y$.

If $x \in\langle 0, y\rangle$ we write $x \leq y$. Analogously, we define $\geq,<$ and $>$ in the obvious way. We say that $x$ is smaller than $y$ if $x<y$. When $x \leq y$ we use the standard notation $[x, y],[x, y),(x, y]$ and $(x, y)$ to denote the closed, half-open and open intervals of endpoints $x$ and $y$.

We mainly use Markov graphs whose vertices are intervals. Namely, if $f \in \mathcal{X}$ and $A$ is a family of intervals of $\mathbb{X}$, the $A$-graph of $f$ is the oriented graph with the intervals of $A$ as vertices and having an arrow from $I$ to $J$ if and only if $I f$-covers $J$. Recall that $I f$-covers $J$ if $f(I) \supset J$ [2, Lemma 1.2.1].

A path of length $k$ in an $A$-graph of $f$ is a sequence of $k+1$ vertices $I_{0}, I_{1}, \ldots, I_{k}$ such that $I_{i-1} f$-covers $I_{i}$ for $i=1, \ldots, k$. We use the obvious representation of such a path: $I_{0} \rightarrow I_{1} \rightarrow \ldots \rightarrow I_{k}$. A path of length 1 will be called a step. A loop of length $k$ in an $A$-graph of $f$ is a path of length $k$ such that $I_{k}=I_{0}$. It will be convenient to consider the indices of the intervals of a loop of length $k$ in $\mathbb{Z}_{k}$. The representations $I_{0} \rightarrow I_{1} \rightarrow \ldots \rightarrow I_{k-1} \rightarrow I_{0}$ and, more generally, $I_{i} \rightarrow I_{i \oplus 1} \rightarrow \ldots \rightarrow I_{i \ominus 1} \rightarrow I_{i}$ are used.

We say that we concatenate the loop $\alpha=I_{0} \rightarrow I_{1} \rightarrow \ldots \rightarrow I_{k-1} \rightarrow I_{0}$ to the loop $\beta=J_{0} \rightarrow J_{1} \rightarrow \ldots \rightarrow J_{l-1} \rightarrow J_{0}$ if they have a common vertex $I_{i}=J_{j}$ and we form a new loop 
$\alpha \beta=I_{0} \rightarrow I_{1} \rightarrow \ldots \rightarrow I_{i} \rightarrow J_{j \oplus_{l} 1} \rightarrow \ldots \rightarrow J_{j} \rightarrow I_{i \oplus_{k} 1} \rightarrow \ldots \rightarrow I_{k-1} \rightarrow I_{0}$.

Usually it will be clear which common vertex we use. The $l$-fold repetition of a loop $\alpha$, denoted by $\alpha^{l}$, is the loop obtained by concatenating $l$ copies of $\alpha$. A loop is nonrepetitive if it is not a repetition of a shorter loop. A loop is elementary if it is not the concatenation of two loops. A shortcut of $s>0$ arrows in a loop $I_{0} \rightarrow I_{1} \rightarrow \ldots \rightarrow I_{k-1} \rightarrow I_{0}$ is any step of the form $I_{i} \rightarrow I_{j}$, with $j \ominus i=s+1$. It is clear that, in such a case, the loop $I_{i} \rightarrow I_{j} \rightarrow I_{j \oplus 1} \rightarrow \ldots \rightarrow I_{i}$ has length $k-s$.

Let $P$ be a periodic orbit of $f \in \mathcal{X}_{n}$. As in the case $n=3$, the ordered pair $A=(x, f(x))$ is an arrow with beginning $b(A)=x$ and end $e(A)=f(x)$. If $b r$ is a branch of $\mathbb{X}$ such that $P \cap b r \neq \emptyset$, we put $\operatorname{sm}(b r)=\min (P \cap b r)$ to represent the smallest point of $P$ on this branch (remember that we do not consider $\{0\}$ as a periodic orbit of $f)$. The arrow beginning at $\operatorname{sm}(b r)$ will be denoted by $\operatorname{sm} A(b r)$, that is, $b(\operatorname{sm} A(b r))=\operatorname{sm}(b r)$. We shall call the arrows $\operatorname{sm} A(b r)$ the smallest arrows.

Let $B$ be the set of branches of $\mathbb{X}$ having nonempty intersection with $P$. We follow [6] to define $\sigma: B \rightarrow B$ such that, for each $b \in B, \sigma(b)=$ $\operatorname{br}(f(\operatorname{sm}(b)))$. Since $B$ is finite, $\sigma$ has at least a periodic point. If $\sigma$ has a periodic point of period $t$, we say that $P$ has type $t$. Note that a periodic orbit can have several types.

Definition 2.1. The periodic orbit $P \subset \mathbb{X}_{n}$ will be called directed if it has type $n$. Observe that if $P$ is directed, then $P$ has points in all branches, $P$ has no other type different from $n$ and $\sigma$ is a cyclic permutation (and, in particular, bijective). The orbit $P$ is undirected if it is not directed.

Definition 2.2. If $P$ is directed and $\mathcal{F}$ is the set of arrows of $P$, we define the colour $c: \mathcal{F} \rightarrow \mathbb{Z}_{n}$ as follows (see Figure 2.1). For each arrow $A \in \mathcal{F}, c(A)$ is such that

$$
\sigma^{c(A)}(\operatorname{br}(e(A)))=\sigma(\operatorname{br}(b(A))) .
$$

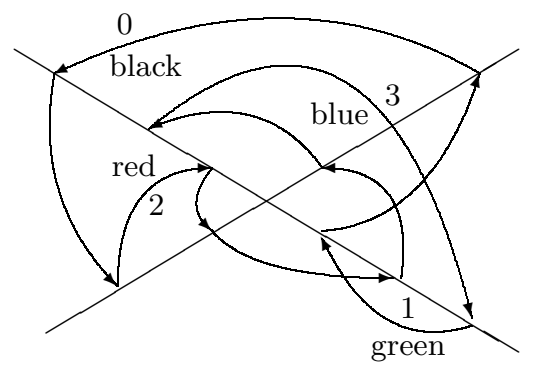

Fig. 2.1. The colours of several arrows of a directed orbit

Note that if $c(A)=0$, then $e(A) \in \sigma(\operatorname{br}(b(A)))$. Hence, for similarity with the case $n=3, A$ will be called black. If $c(A) \neq 0$, then $A$ will be 
called coloured. Observe that, by definition, all smallest arrows are black. Recall that, in the case $n=3$, an arrow $A$ was called green if $c(A)=1$ and red when $c(A)=2$. For $n=4$ we keep this terminology and add the term blue for arrows with $c(A)=3$.

Analogously we define the colour of a step $I \rightarrow J$ in any $A$-graph of $f$. Namely, if $x \in I$ and $y \in J$, then the colour of $I \rightarrow J$ is the number $c \in \mathbb{Z}_{n}$ such that $\sigma^{c}(\operatorname{br}(y))=\sigma(\operatorname{br}(x))$.

Now we introduce a new notion which will play a key role in our study.

Definition 2.3. Let $P$ be a directed orbit of a map $f \in \mathcal{X}_{n}$. Then $S \subset P$ is a string of $P$ of length $l(S)=\operatorname{Card}(S)$ if it satisfies the following:

(i) there is a point $z \in S$, called the beginning of $S$, such that $S=$ $\left\{f^{k}(z): 0 \leq k<l(S)\right\}$

(ii) if $0<k<l(S)$, then the arrow $\left(f^{k-1}(z), f^{k}(z)\right)$ is black, and

(iii) if an endpoint of a black arrow is in $S$, then so is the other endpoint.

The point $f^{l(S)-1}(z)$ will be called the end of $S$ and the string beginning at $f^{l(S)}(z)$ will be called the next string of $S$.

Observe that, if $P$ has only black arrows, then we can take any point as the beginning of the unique existing string (the entire orbit). Otherwise, the beginning of a string is the end of a coloured arrow, and the end of a string is the beginning of a coloured arrow. In particular, if $A$ and $B$ are coloured arrows such that $e(A)=b(B)=x$, then $S=\{x\}$ is a string. Each coloured arrow separates the string ending in the beginning of the arrow from the next string. Note that these two strings can be the same. Then it is clear that the set of strings of $P$ forms a partition of $P$ determined by the coloured arrows, if there are any. Therefore, except in the special case of a periodic orbit with no coloured arrows, the numbers of strings and of coloured arrows coincide.

We say that a string $S$ spirals out if $x<f^{n}(x)$ whenever $f^{k}(x) \in S$ for $k=0,1, \ldots, n$. Note that if $P$ has only black arrows and the period of $P$ is larger than $n$, then the string $P$ does not spiral out.

We shall often label the branches of $\mathbb{X}_{n}$ as $b r_{0}, b r_{1}, \ldots, b r_{n-1}$. Then we can simplify the notation in the following way: if $b r_{i} \cap P \neq \emptyset$, we put $s m_{i}=$ $\operatorname{sm}\left(b r_{i}\right)$ and $s m A_{i}=\operatorname{sm} A\left(b r_{i}\right)$. In that case we will also define the index of $x \in \mathbb{X}_{n} \backslash E$ in such a way that $\operatorname{ind}(x)=i$ if and only if $x \in b r_{i}$.

If $P$ is directed then we can label the branches of $\mathbb{X}_{n}$ in such a way that $\sigma\left(b r_{i}\right)=b r_{i \oplus 1}$ for each $i \in \mathbb{Z}_{n}$. In this case, we shall say that the branches of $\mathbb{X}_{n}$ are $\sigma$-labelled. Then $\operatorname{ind}\left(e\left(s m A_{i}\right)\right)=\operatorname{ind}\left(b\left(s m A_{i}\right)\right) \oplus 1$ for $i \in \mathbb{Z}_{n}$ and, for every arrow $A$, it follows that

$$
\operatorname{ind}(e(A))+c(A) \equiv \operatorname{ind}(b(A))+1,
$$

from the definition of $c(A)$. 
Lemma 2.4. Let $P$ be directed and assume that the branches of $\mathbb{X}_{n}$ are $\sigma$-labelled. If $A_{0}, A_{1}, \ldots, A_{k-1}$ is a sequence of $k$ arrows of $P$ such that $b\left(A_{j}\right) \sim e\left(A_{j-1}\right)$ for $j=1, \ldots, k-1$, then

$$
\operatorname{ind}\left(e\left(A_{k-1}\right)\right)+\sum_{j=0}^{k-1} c\left(A_{j}\right) \equiv \operatorname{ind}\left(b\left(A_{0}\right)\right)+k .
$$

Proof. This follows by adding up the congruences (1) for these $k$ arrows.

The sequences of arrows defined next will play a basic role in the description of the orbits we are going to study in this work.

Definition 2.5. The $k \geq 1$ arrows $A_{0}, A_{1}, \ldots, A_{k-1}$ are said to be overlapping if $e\left(A_{i}\right) \geq b\left(A_{i \oplus 1}\right)$ for all $i \in \mathbb{Z}_{k}$.

Lemma 2.6. If $A_{0}, A_{1}, \ldots, A_{k-1}$ are overlapping arrows of a directed orbit, then

$$
k \equiv \sum_{i=0}^{k-1} c\left(A_{i}\right)
$$

Proof. This follows immediately from Lemma 2.4.

From now on we assume that $P$ is a directed periodic orbit of period $m$ of a map $f \in \mathcal{X}_{n}$. Therefore, $m \geq n$. Since our aim is to characterize the primary orbits, in view of the First Theorem (Theorem 2.3) of [1], we can (and will) also assume that $f$ is $E P$-adjusted.

Clearly, the $n$ smallest arrows are overlapping and give a loop of length $n$ in the EP-graph of $f$ which will be called the branching loop. Namely, if we assume that the branches of $\mathbb{X}_{n}$ are $\sigma$-labelled, for $i=0,1, \ldots, n-1$ we define $I_{i}=\left[0, s m_{i}\right]$ to be the branching intervals. Then the branching loop is $\alpha=I_{0} \rightarrow I_{1} \rightarrow \ldots \rightarrow I_{n-1} \rightarrow I_{0}$.

Let $A_{0}, A_{1}, \ldots, A_{k-1}$ be a sequence of arrows and let $C_{0}, C_{1}, \ldots, C_{l-1}$ be the sequence of coloured arrows in it. Then $C_{i}=A_{j_{i}}$ for $i=0,1, \ldots, l-1$. We will always assume that $j_{i}<j_{k}$ if and only if $i<k$.

Definition 2.7. Let $\Gamma=\left(A_{0}, A_{1}, \ldots, A_{k-1}\right)$ and $\Gamma^{\prime}=\left(A_{0}^{\prime}, A_{1}^{\prime}, \ldots, A_{k^{\prime}-1}^{\prime}\right)$ be two sequences of arrows. Let $C_{0}, C_{1}, \ldots, C_{l-1}$ and $C_{0}^{\prime}, C_{1}^{\prime}, \ldots, C_{l^{\prime}-1}^{\prime}$ be the sequences of coloured arrows of the above sequences, respectively. We say that $\Gamma$ and $\Gamma^{\prime}$ are isochromatic if $l=l^{\prime}$ and there exists a $j \in \mathbb{Z}_{l}$ such that $c\left(C_{i}\right)=c\left(C_{j \oplus i}^{\prime}\right)$ for all $i \in \mathbb{Z}_{l}$. If $Q$ is a directed periodic orbit of period $p$,

$$
\Gamma^{\prime}=\left((x, f(x)),\left(f(x), f^{2}(x)\right), \ldots,\left(f^{p-1}(x), x\right)\right)
$$

is the sequence of arrows of $Q$ and $\Gamma$ and $\Gamma^{\prime}$ are isochromatic, then we also say that $\Gamma$ and $Q$ are isochromatic. 
The following lemma and its corollary will be useful to obtain orbits (different from $P$ ) from a sequence of overlapping arrows.

Lemma 2.8. Let $P$ be a directed orbit of an EP-adjusted map $f \in \mathcal{X}_{n}$. Let $k \not \equiv 0$ and let $\Gamma=\left(A_{0}, A_{1}, \ldots, A_{k-1}\right)$ be a sequence of overlapping arrows, with $A_{0}$ being the smallest arrow on its branch. Then, for every $l \geq 1, f$ has a directed orbit $Q \neq P$ of period $k+\ln$ such that $\Gamma$ and $Q$ are isochromatic.

Proof. Since $A_{0}$ is the smallest arrow on its branch, it is black. Hence $k>1$. Since $k \not \equiv 0$ and $b\left(A_{i \oplus 1}\right) \leq e\left(A_{i}\right)$ for each $i \in \mathbb{Z}_{k}$, by Lemma 2.6 there is at least one coloured arrow $A_{p}$ (with $p \neq 0$ ).

For $i \in \mathbb{Z}_{k}$ we define the intervals $J_{n+i}$ as follows:

$$
\begin{aligned}
& J_{n+i}=\left[0, b\left(A_{i}\right)\right] \quad \text { if } i \neq p, \\
& J_{n+p}=\left[\operatorname{sm}\left(\operatorname{br}\left(b\left(A_{p}\right)\right)\right), b\left(A_{p}\right)\right] .
\end{aligned}
$$

Then $J_{n} \rightarrow J_{n+1} \rightarrow \ldots \rightarrow J_{n+k-1} \rightarrow J_{n}$. Hence, since $J_{n}$ is a basic interval, by [1, Lemma 1.12], for each $i \in \mathbb{Z}_{k}$ there exists a basic interval $I_{n+i} \subset J_{n+i}$ such that $I_{n} \rightarrow I_{n+1} \rightarrow \ldots \rightarrow I_{n+k-1} \rightarrow I_{n}$ (in fact $J_{n}=I_{n}$ ). We call this loop $\beta$. By construction, the $i$ th step in $\beta\left(I_{n+i} \rightarrow I_{n+(i \oplus 1)}\right)$ is of the same colour as $A_{i}$ for all $i \in \mathbb{Z}_{k}$.

If $\alpha=I_{0} \rightarrow I_{1} \rightarrow \ldots \rightarrow I_{n-1} \rightarrow I_{0}$ is the branching loop, with $I_{0}=I_{n}$, we can consider the loop $\gamma=\alpha^{l} \beta$. If $\gamma$ is repetitive, since $\alpha$ is elementary, then $\beta=\delta \alpha \varepsilon$ for some (possibly empty) loops $\delta$ and $\varepsilon$ starting with $I_{0}$. Then we can consider the loop $\alpha^{l+1} \delta \varepsilon$ which has the same steps as $\gamma$, but ordered in a different way. Nevertheless, since all the steps in $\alpha$ are black, this loop has the same coloured steps as $\gamma$ and these steps are arranged in the same order. Repeating this process if necessary, since $k \not \equiv 0$, we get a nonrepetitive loop $\gamma^{\prime}=\alpha^{r} \beta^{\prime}$ for some $r \geq l$ and some nonempty loop $\beta^{\prime}$ of length not a multiple of $n$ (if $\gamma$ is nonrepetitive, then we take $\gamma^{\prime}=\gamma$ ). All the coloured steps of $\gamma$ are now in $\beta^{\prime}$, arranged in the same order.

Since $0 \notin I_{n+p}$ and $\gamma^{\prime}$ goes through $I_{n+p}$, by [6, Lemma 2.2], we obtain a periodic orbit $Q$ of period $k+\ln$ isochromatic with $\Gamma$.

Clearly $P \neq Q$ because $\gamma^{\prime}$ goes at least twice through $I_{0}$ but $P$ has a unique point in $I_{0}$. It is also clear that $Q$ is directed. Indeed, all the smallest arrows of $Q$ must begin in the branching EP-basic intervals, and all the steps of the $E P$-graph of $f$ starting from these intervals are black.

Corollary 2.9. Let $A_{0}, A_{1}, \ldots, A_{q-1}$ be a sequence of arrows such that $A_{0}$ is the smallest arrow on its branch, $e\left(A_{i}\right) \geq b\left(A_{i+1}\right)$ for $i=0,1, \ldots, q-2$ and $\sum_{i=0}^{q-1} c\left(A_{i}\right) \not \equiv 0$. Then $f$ has directed orbits of all the periods congruent to $\sum_{i=0}^{q-1} c\left(A_{i}\right)$ and greater than or equal to $q+n$. Moreover, these orbits are isochromatic with the sequence $A_{0}, A_{1}, \ldots, A_{q-1}$. 
Proof. We label the branches in such a way that ind $\left(e\left(A_{q-1}\right)\right)=0$ and they are $\sigma$-labelled. Let $r=\operatorname{ind}\left(b\left(A_{0}\right)\right)$. Then we have the $q+r$ overlapping arrows

$$
A_{0}, A_{1}, \ldots, A_{q-1}, s m A_{0}, \ldots, s m A_{r-1},
$$

where the last $r$ arrows are omitted if $r=0$. By Lemma 2.6, $q+r \equiv$ $\sum_{i=0}^{q-1} c\left(A_{i}\right)$. Then Lemma 2.8 (with $k=q+r$ ) ends the proof.

The first important consequence of Lemma 2.8 is the following property of primary directed orbits.

Proposition 2.10. Each string of a primary directed orbit of a map from $\mathcal{X}_{n}$ with period not a multiple of $n$ spirals out.

Proof. Let $P$ be a primary directed orbit of $f \in \mathcal{X}_{n}$ of period $m \neq 0$ and let $S$ be a string of $P$. Assume that there is some $x \in S$ with $f^{j}(x) \in S$ for $j=0,1, \ldots, n$ and such that $x>f^{n}(x)$. Then we label the points and arrows of $P$ as follows:

$$
x_{0}=\operatorname{sm}(\operatorname{br}(x)) \quad \text { and } \quad x_{i}=b\left(A_{i}\right)=f^{i}\left(x_{0}\right) \quad \text { for each } i \in \mathbb{Z}_{m} .
$$

Since $x \neq x_{0}$, there is an $l>0$ (in fact, $l>1$, because $A_{0}$ is black) such that $x=x_{l}$. Moreover, since $x_{l \oplus j}=f^{j}(x) \in S$ for $j=0,1, \ldots, n$, we have $f^{j}(x) \not x_{0}$ for $j=1, \ldots, n-1$. That is, $x_{l \oplus j} \neq x_{0}$ for $j=0,1, \ldots, n-1$ and, hence, $l+n \leq m$.

Then, by eliminating the arrows $A_{l}, A_{l+1}, \ldots, A_{l+n-1}$ from the sequence of arrows of $P$, we get the sequence

$$
A_{0}, A_{1}, \ldots, A_{l-1}, A_{l+n}, \ldots, A_{m-1}
$$

(where the arrows $A_{l+n}, \ldots, A_{m-1}$ are omitted if $l+n=m$ ) of $k=m-n$ arrows. These arrows are overlapping because $e\left(A_{l-1}\right)=x>f^{n}(x)=$ $x_{l \oplus n}=b\left(A_{l \oplus n}\right)$. Since $A_{0}=\operatorname{sm} A(\operatorname{br}(x))$ (and it has not been eliminated), Lemma 2.8 gives a periodic orbit of period $k+n=m$ different from $P$. By the First Theorem (Theorem 2.3) of [1], this contradicts the primarity of $P$ (remember that we are assuming that $f$ is $E P$-adjusted).

3. Twist and single orbits for maps from $\mathcal{X}_{n}$. We are ready to study the simplest families of primary directed orbits. Namely, those having at most one coloured arrow. This study can be done for every $\mathcal{X}_{n}(n \geq 2)$ by generalizing the analogous results of [1].

First we assume that $P$ is a directed periodic orbit of period $m$ of a map $f \in \mathcal{X}_{n}$, with all arrows black. Note that, then, $m \equiv 0$ by Lemma 2.6. The characterization of the primary orbits of this kind is a routine generalization of the corresponding results for $\mathcal{Y}$ maps and, hence, we omit its proof. This generalization of [1, Definition 4.40, Proposition 5.7 and Directed Theorem (a)] is as follows. 
Definition 3.1. We say that $P$ is a twist orbit in the following cases:

(1) If $m=n \cdot 2^{k}, k \geq 0$, we use induction. If $k=0$ then $P$ is twist. If $k \geq 1$ and twist orbits of period $n \cdot 2^{k-1}$ are defined, then $P$ is twist if it is a 2 -extension of a twist orbit of period $n \cdot 2^{k-1}$.

(2) If $m=n \cdot 2^{k} \cdot r, r$ is odd and $r \geq 3$, then $P$ is twist if it is an $R$-extension of a twist orbit of period $n \cdot 2^{k}$, where $R$ is a simple orbit of period $r$.

THEOREM 3.2. If $P$ is a directed orbit having only black arrows, then $P$ is primary if and only if it is twist.

We now study the primary directed orbits having only one coloured arrow. The generalization of the related results of [1] to any $\mathcal{X}_{n}(n \geq 2)$ can also be done in a simple way. We shall see that the orbits of this kind are defined as follows.

Definition 3.3. Let $P$ be a directed orbit of a map $f \in \mathcal{X}_{n}$. $P$ will be called a single orbit of colour $c$ if it has exactly one coloured arrow of colour $c$ and its unique string spirals out.

If $P$ is a single orbit of colour $c$ and period $m$ of a map $f \in \mathcal{X}_{n}$, then $m \equiv c$ by Lemma 2.6, and $m>n$ because $P$ is directed and has a coloured arrow. If $A$ is this coloured arrow, we set $x_{i}=f^{i}(e(A))$ for $i \in \mathbb{Z}_{m}$. Then we have $x_{i}<x_{i+n}$ for $0 \leq i \leq m-n-1$.

Note that if the branches of $\mathbb{X}_{n}$ are labelled in such a way that $x_{j} \in b r_{j}$ for $j \in \mathbb{Z}_{n}$, then $x_{i} \in b r_{j}$ is equivalent to $i \equiv j$. That is, $\operatorname{ind}\left(x_{i}\right) \equiv i$ for each $i \in \mathbb{Z}_{m}$. Then it also happens that $x_{j}=s m_{j}$ for $j \in \mathbb{Z}_{n}$. We shall label the $E P$-basic intervals by their largest endpoint, that is, $I_{i}=\left[0, x_{i}\right]$ if $0 \leq i<n$ and $I_{i}=\left[x_{i-n}, x_{i}\right]$ if $n \leq i \leq m-1$.

By Proposition 2.10, a directed primary orbit with only one coloured arrow must be single. To see that the converse is also true we start by characterizing the EP-graph of a single orbit. The following results are closely related to $[6$, Theorem 3.2$]$.

LEMMA 3.4. Let $P$ be a single orbit of an EP-adjusted map $f \in \mathcal{X}_{n}$. Then the EP-graph of $f$ contains only the following steps (see Figure 3.1):

(i) $I_{i} \rightarrow I_{i \oplus 1}$ for each $i \in \mathbb{Z}_{m}$.

(ii) $I_{n-1} \rightarrow I_{0}$.

(iii) $I_{m-1} \rightarrow I_{i}$ for all $i \equiv m$.

Proof. Let $i \in \mathbb{Z}_{m}$ be such that $0 \leq i<m-1$. Since $f$ is $E P$-adjusted, if $I_{i}=\left[x, x_{i}\right]$ with $x \in E P$, then $f\left(I_{i}\right)=\left[f(x), f\left(x_{i}\right)\right]$. If $i \neq n-1$, then $f\left(I_{i}\right)=I_{i+1}$. Since $f(0)=0<x_{0}<x_{n}=f\left(x_{n-1}\right)$, we have $f\left(I_{n-1}\right)=$ $I_{0} \cup I_{n}$. 

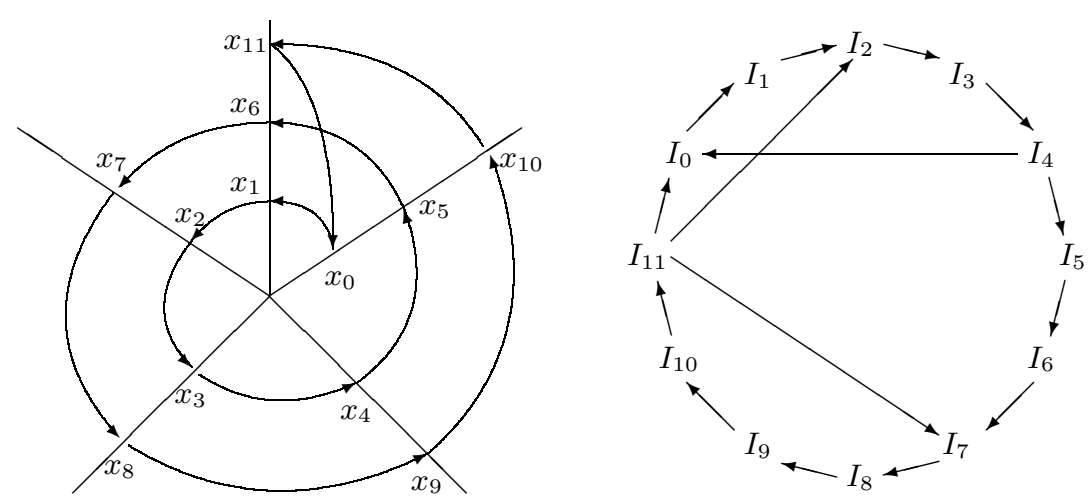

Fig. 3.1. A single orbit $P$ and the $E P$-graph of an EP-adjusted map

On the other hand, since $I_{m-1}=\left[x_{m-1-n}, x_{m-1}\right]$ and $f\left(x_{m-n-1}\right)=$ $x_{m-n} \not x_{0}=f\left(x_{m-1}\right)$, we have $f\left(I_{m-1}\right)=I_{0} \cup\left[0, x_{m-n}\right]$ (we also use the fact that $f$ is $E P$-adjusted). By taking into account that $0<x_{i} \leq x_{m-n}$ if and only if $i \equiv m$, the lemma is proved.

The steps in (i) of the above lemma form the loop $I_{0} \rightarrow I_{1} \rightarrow \ldots \rightarrow$ $I_{m-1} \rightarrow I_{0}$ which will be called the fundamental loop.

Corollary 3.5. Let $P$ be a single orbit of an EP-adjusted map $f \in \mathcal{X}_{n}$. Then every elementary loop in the EP-graph of $f$ different from the fundamental one has length a multiple of $n$.

Proof. The shortcut given in (ii) of Lemma 3.4 produces a branching loop, which has length $n$. The shortcuts of $i$ arrows given in (iii) of Lemma 3.4 produce elementary loops of lengths $m-i \equiv 0$. Since, in view of Lemma 3.4, there are no more elementary loops different from the fundamental one in the EP-graph of $f$, the corollary follows.

Finally we are ready to prove the desired result.

THEOREM 3.6. Let $P$ be a directed orbit having only one coloured arrow $A$. Then $P$ is primary if and only if it is single of colour $c(A)$.

Proof. As already mentioned, if $P$ is primary then it must be single by Proposition 2.10. Therefore we only have to prove the converse. Let $P$ be a single orbit (of colour $c(A)$, of course) of period $m$ and let $f \in \mathcal{X}_{n}$ be $E P$-adjusted. In the EP-graph of $f$ there is no thin loop. Indeed, by Lemma 3.4 all the loops go through $I_{n-1}$ or through $I_{m-1}$ which $f$-cover more than one basic interval. By Corollary 3.5 the only loop of length $m$ is the fundamental one, which is nonrepetitive and is associated to $P$. Hence, by the First Theorem of [1], $P$ is primary.

The next lemma shows that, with a single orbit, there coexist many other orbits. This result, which will be useful later, is related to the "forcing 
relation" (see [2]) and the characterization of the sets of periods of maps from $\mathcal{X}_{n}$ (see $\left.[6]\right)$.

Lemma 3.7. Assume that $P$ is a single orbit of colour $c$ and period $m$ of an EP-adjusted map $f \in \mathcal{X}_{n}$. Then $f$ has:

(a) single orbits of colour $c$ and periods $m+\ln$ for all $l \geq 1$;

(b) periodic orbits of periods $k m+\ln$ with $k$ arrows of colour $c$, for all $k, l \geq 1$

(c) twist orbits of all periods $k \equiv 0$.

All these orbits have spans strictly contained in $\langle P\rangle$. Moreover, $f$ has no other periodic orbits different from $P$.

Proof. The proofs of (a)-(c) are straightforward generalizations of [1, Lemmas 10.7 and 10.14]. The fact that the spans of those orbits are strictly contained in $\langle P\rangle$ follows easily from their constructions.

By Corollary 3.5, in the EP-graph of $f$ we can only find nonrepetitive loops of lengths $k m+l n$, with $k \geq 0$ and $l \geq 1$, different from the fundamental one. Hence, the last statement of the lemma follows from [1, Proposition 1.11] and the primarity of $P$.

4. Strongly directed orbits of maps of $\mathcal{X}_{n}$. In this section we shall define and study a new class of periodic orbits, more restrictive than the directed ones, that plays the same role as the directed orbits of maps from $\mathcal{Y}$ in the classification of all primary orbits of maps from $\mathcal{X}_{n}$. We shall explain this point in detail later. The definition of this new class will be done by means of the following property.

Generalized Directed Rule. Let $P$ be a periodic orbit of a map $f \in \mathcal{X}_{n}$. For each sequence $A_{0}, A_{1}, \ldots, A_{k-1}$ of overlapping arrows of $P$, we have $k \geq n$.

Definition 4.1. A directed periodic orbit $P$ will be called strongly directed if it satisfies the Generalized Directed Rule.

Obviously, a directed orbit with only black arrows is strongly directed. Clearly, also single orbits are strongly directed.

REMARK 4.2. In [1, Lemmas 6.1 and 10.4] it is shown that, for $n \leq 3$, all the primary directed orbits have the following properties:

(i) There is no arrow $A$ such that $b(A)<e(A)$.

(ii) If $n=3$ then there are no arrows $A$ and $B$ such that $e(A) \geq b(B)$ and $e(B) \geq b(A)$.

Clearly, the Generalized Directed Rule reduces to the above properties for $n \leq 3$. Therefore, all primary directed orbits for $n \leq 3$ are also strongly 
directed. However, despite the fact that each primary directed orbit satisfies (i) for all $n \geq 2$ (see the proof of [1, Lemma 6.1]), for $n \geq 4$ we can easily find primary directed orbits which are not strongly directed. To see this, consider the following example.

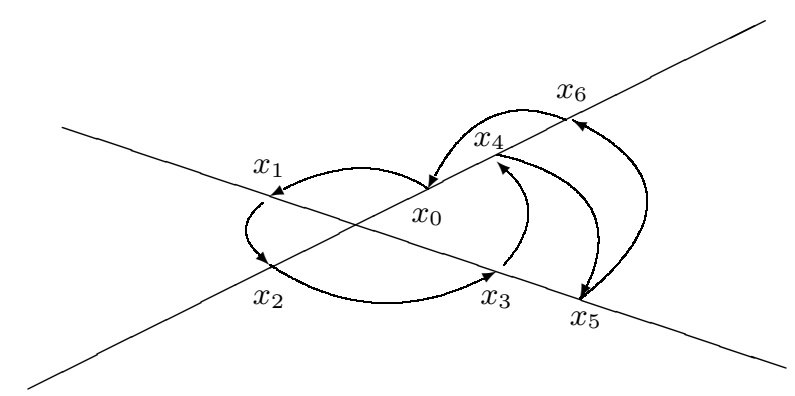

Fig. 4.1. A primary directed orbit which is not strongly directed

Assume that $P$ is the directed periodic orbit of an $E P$-adjusted map $f \in \mathcal{X}_{4}$ of period 7 described as follows (see Figure 4.1). The points and arrows of $P$ are labelled in the natural way, that is, $f\left(x_{i}\right)=x_{i \oplus 1}$ and $b\left(A_{i}\right)=x_{i}$ for all $i \in \mathbb{Z}_{7}$. Furthermore, for $j \in \mathbb{Z}_{4}, x_{j}$ is the smallest point on its branch. Since the arrows $A_{j}$ have to be black for $j \in \mathbb{Z}_{4}$, we have $x_{0}<x_{4}$. Finally, we set $x_{3}<x_{5}$ and $x_{4}<x_{6}$. Clearly this orbit is not strongly directed, because the arrows $A_{3}$ (black) and $A_{4}$ (red) are overlapping. However, it is very easy to see that $P$ is primary. Indeed, in the EP-graph of $f$ there is only one loop of length 7 , which is elementary and is associated to $P$. By [1, Proposition 1.10(b)] there is no periodic orbit different from $P$ associated to this loop. All other elementary loops in that graph have even length ( 2 or 4$)$. Hence, we cannot get any periodic orbit of period 7 different from $P$, and $P$ is primary by the First Theorem of [1].

For strongly directed orbits we have the following couple of results about overlapping arrows, which we shall need later.

Lemma 4.3. Let $P$ be a strongly directed orbit and let $A_{0}, A_{1}, \ldots, A_{k-1}$ be overlapping arrows. If there are $s$ consecutive arrows, $A_{i}, A_{i \oplus 1}, \ldots$ $\ldots, A_{i \oplus(s-1)}$, in the above sequence such that $s \equiv \sum_{j=0}^{s-1} c\left(A_{i \oplus j}\right)$ and $s-$ $\operatorname{Card}\left(\left\{0 \leq j \leq s-1: c\left(A_{i \oplus j}\right)=1\right\}\right)<n$, then $A_{i \oplus s}, A_{i \oplus(s+1)}, \ldots, A_{i \ominus 1}$ are overlapping arrows.

Proof. We only have to prove that $e\left(A_{i \ominus 1}\right) \geq b\left(A_{i \oplus s}\right)$. Since $e\left(A_{i \ominus 1}\right)$ $\geq b\left(A_{i}\right)$ and $e\left(A_{i \oplus(s-1)}\right) \geq b\left(A_{i \oplus s}\right)$, it is enough to show that $b\left(A_{i}\right) \geq$ $e\left(A_{i \oplus(s-1)}\right)$.

First, we prove that $b\left(A_{i}\right) \sim e\left(A_{i \oplus(s-1)}\right)$. Indeed, if we assume that the branches of $\mathbb{X}_{n}$ are $\sigma$-labelled, then 


$$
\operatorname{ind}\left(e\left(A_{i \oplus(s-1)}\right)\right)+\sum_{j=0}^{s-1} c\left(A_{i \oplus j}\right) \equiv \operatorname{ind}\left(b\left(A_{i}\right)\right)+s,
$$

by Lemma 2.4. Since $s \equiv \sum_{j=0}^{s-1} c\left(A_{i \oplus j}\right)$ we get $b\left(A_{i}\right) \sim e\left(A_{i \oplus(s-1)}\right)$.

In the case $s=1$ the Generalized Directed Rule prohibits that $b\left(A_{i}\right)<$ $e\left(A_{i}\right)$, and thus the lemma is proved for this case.

Furthermore, the previous case allows us to eliminate the arrows $A_{i \oplus j}$ with $0 \leq j \leq s-1$ such that $c\left(A_{i \oplus j}\right)=1$ from the sequence $A_{0}, A_{1}, \ldots, A_{k-1}$. Thus, it only remains to consider the case $1<s<n$. Then, since $e\left(A_{i \oplus j}\right) \geq$ $b\left(A_{i \oplus(j+1)}\right)$ for $j=0,1, \ldots, s-2$, again by the Generalized Directed Rule it cannot happen that $e\left(A_{i \oplus(s-1)}\right) \geq b\left(A_{i}\right)$.

Lemma 4.4. Let $P$ be a strongly directed orbit and let $A_{0}, A_{1}, \ldots, A_{k-1}$ be overlapping arrows, with $A_{0}$ being the smallest arrow on its branch. Let $j \in\{1, \ldots, k-1\}$ be such that $A_{0}, A_{1}, \ldots, A_{j-1}$ are black arrows. Then $j \geq c\left(A_{j}\right)$ and $A_{0}, \ldots, A_{j-c}, A_{j+1}, \ldots, A_{k-1}$ are overlapping arrows.

Proof. Assume that the branches of $\mathbb{X}_{n}$ are $\sigma$-labelled in such a way that $\operatorname{ind}\left(e\left(A_{j}\right)\right)=0$. Since $A_{0}, A_{1}, \ldots, A_{k-1}$ are overlapping and $A_{0}, A_{1}, \ldots, A_{j-1}$ are black arrows, by Lemma 2.4 , we have ind $\left(b\left(A_{0}\right)\right) \equiv c-(j+1)$, where $c=$ $c\left(A_{j}\right)$. If $j<c$, then $0 \leq c-(j+1)<n$ and, hence, $\operatorname{ind}\left(b\left(A_{0}\right)\right)=c-(j+1)$. Since $e\left(A_{j}\right) \geq s m_{0}$, the $c$ arrows

$$
A_{0}, A_{1}, \ldots, A_{j-1}, A_{j}, s m A_{0}, \ldots, s m A_{c-j-2}
$$

(where the last $c-j-1$ arrows are omitted if $c<j+2$ ) are overlapping, contrary to the Generalized Directed Rule. Therefore, $j \geq c$.

The last statement follows then from Lemma 4.3 with $i=j-c+1$ and $s=c$.

The auxiliary results of $[1$, Section 10] about directed orbits of $\mathcal{Y}$ maps which depend on the Directed Rule can easily be extended to $\mathbb{X}_{n}$ for strongly directed orbits. In particular, we shall use the following versions of $[1$, Lemmas 10.8 and 10.10].

Lemma 4.5. Let $P$ be a strongly directed orbit of $f \in \mathcal{X}_{n}$. Let $A_{0}, A_{1}, \ldots$ $\ldots, A_{k-1}$ be overlapping arrows, all black except $A_{0}$ which is coloured. Then $f$ has a single orbit of period $k$ and colour $c\left(A_{0}\right)$, with span contained in $\langle P\rangle$.

Lemma 4.6. Let $P$ be a strongly directed orbit of period $m$ of an EPadjusted map $f \in \mathcal{X}_{n}$. If $P$ has a coloured arrow of colour $c$, then $f$ has a periodic orbit of period smaller than or equal to $m$ which is single of colour $c$.

We also have the following important result which, generalizing [1, Corollary 10.11], characterizes the primary strongly directed orbits whose periods satisfy a certain congruence. 
Proposition 4.7. Let $P$ be strongly directed and primary of period $m$. Then:

(a) If $m \equiv 0$, then all arrows of $P$ are black and, hence, $P$ is twist.

(b) If $A$ is a coloured arrow of $P$ and $m \equiv c(A)$, then $P$ is single of colour $c(A)$.

Proof. Let $f \in \mathcal{X}_{n}$ be an EP-adjusted map.

(a) If there is a coloured arrow, then by Lemmas 4.6, 3.7(c) and [1, Lemma 1.18], $f$ has an orbit of period $m$ with all arrows black. Hence it is different from $P$. But by the First Theorem of [1], this contradicts the primarity of $P$.

(b) By Lemmas 4.6, 3.7(a) and [1, Lemma 1.18], $f$ has a single orbit of period $m$. Then $P$ must be single, again by the First Theorem of [1].

From the above proposition we get immediately the following generalization of $[1$, Directed Theorem (b)].

Corollary 4.8. If $n>2$ and $P$ is primary and strongly directed, then $P$ cannot have arrows of all colours.

At present we know how the primary strongly directed orbits look like if they have no coloured arrows (twist) or if they have just one coloured arrow (single). In the rest of this section we are going to show that, in the case $n=4$, such orbits cannot have more than three coloured arrows. This will help us in the rest of our study.

We start by proving a lemma whose proof we owe to the kindness of J. Moncasi and his colleagues.

LEMma 4.9 (Moncasi). If $n \geq 2$ and $a_{0}, a_{1}, \ldots, a_{n-1} \in \mathbb{Z}_{n} \backslash\{0\}$, then there exist $i, j \in \mathbb{Z}_{n}, i<j$, such that $\sum_{k=i}^{j} a_{k} \equiv 0$.

Proof. Set $s_{j}=\sum_{k=0}^{j} a_{k}$ for $j \in \mathbb{Z}_{n}$. If $s_{j} \equiv 0$ for some $j \in \mathbb{Z}_{n}$, then, since $a_{0} \neq 0$, we have $j>0$ and we are done.

If $s_{j} \not \equiv 0$ for all $j \in \mathbb{Z}_{n}$, then since $\mathbb{Z}_{n} \backslash\{0\}$ has only $n-1$ elements, we can find $i, j \in \mathbb{Z}_{n} \backslash\{0\}$ with $i \leq j$ such that $s_{i-1} \equiv s_{j}$; that is, $s_{j}-s_{i-1}=$ $a_{i}+\ldots+a_{j} \equiv 0$. Furthermore, $i \neq j$ since $a_{i} \neq 0$ for all $i \in \mathbb{Z}_{n}$.

The following theorem plays a crucial role in the characterization of the primary strongly directed orbits of maps of $\mathcal{X}_{4}$.

THEOREM 4.10. If $n=4$ and $P$ is strongly directed and primary, then it cannot have more than three coloured arrows.

Proof. Label the arrows $A_{0}, A_{1}, \ldots, A_{m-1}$ of $P$ in such a way that $A_{0}$ is the smallest arrow on its branch, and $b\left(A_{i}\right)=e\left(A_{i-1}\right)$ for $i=1, \ldots, m-1$. Let $C_{0}, C_{1}, \ldots, C_{l-1}$ be the sequence of coloured arrows in the above sequence. Recall that, if $C_{i}=A_{j_{i}}$ for $i \in \mathbb{Z}_{l}$, we assume that $j_{i}<j_{k}$ if and 
only if $i<k$. Then, for each $i=0,1, \ldots, l-1$, we denote by $n(i)$ the number of black arrows of the sequence $A_{0}, A_{1}, \ldots, A_{m-1}$ before $C_{i}$. That is, $n(i)=j_{i}-i$.

We assume that $l \geq n$ and we shall get a contradiction. Note first that $m \not \equiv 0$ by Proposition 4.7(a). Also, by Lemma 4.9, there are indices $p, q \in \mathbb{Z}_{n}$ with $p<q$ such that $\sum_{i=p}^{q} c\left(C_{i}\right) \equiv 0$. Let $p$ be the smallest such index.

If $p=0$, then we can apply Lemma 4.4 the necessary number of times $(q+1)$ to eliminate the arrows $C_{0}, C_{1}, \ldots, C_{q}$, together with $\sum_{i=0}^{q} c\left(C_{i}\right)-$ $(q+1)$ black arrows. In this way we obtain $m-\sum_{i=0}^{q} c\left(C_{i}\right)$ overlapping arrows, beginning with $A_{0}$. Thus, since $m-\sum_{i=0}^{q} c\left(C_{i}\right) \equiv m$, we can apply Lemma 2.8 to get a periodic orbit of period $m$ different from $P$. This contradicts the primarity of $P$ by the First Theorem of [1].

Now suppose that $p>0$. We can assume that, in the sequence of arrows $A_{0}, A_{1}, \ldots, A_{m-1}$, there is no smallest arrow between $C_{0}$ and $C_{1}$. Indeed, if there were one, relabelling the arrows from that one on, we could take a smaller $p$. Relabelling if necessary, we can also assume that there is no smallest arrow between $A_{0}$ and $C_{0}$ other than $A_{0}$. Under these assumptions, we have $n(1)+2 \leq m-(n-1)-(l-2)$.

We note that if $m \equiv c\left(C_{0}\right)+c\left(C_{1}\right)$, then by using Corollary 2.9 with $q=n(1)+2$, we get a periodic orbit of period $m$ (because $n(1)+2+n<m$ ) with only two coloured arrows. Hence, this orbit is different from $P$, which contradicts the primarity of $P$.

Since $p>0$, it is clear that $c\left(C_{0}\right) \oplus c\left(C_{1}\right) \in \mathbb{Z}_{4} \backslash\left\{0, c\left(C_{0}\right), c\left(C_{1}\right)\right\}$. Also $m \not \equiv c\left(C_{0}\right)$ and $m \not \equiv c\left(C_{1}\right)$ by Proposition $4.7(\mathrm{~b})$. Therefore, since $m \not \equiv 0$, if $c\left(C_{0}\right) \neq c\left(C_{1}\right)$ we get $m \equiv c\left(C_{0}\right)+c\left(C_{1}\right)$, which gives a contradiction.

So we are left with the case $c\left(C_{0}\right)=c\left(C_{1}\right)=c$. Clearly, $c \neq 2$ (that is, $c \in\{1,3\}$ ). If $P$ has no coloured arrow of colour 2 , then again $m \equiv$ $c\left(C_{0}\right)+c\left(C_{1}\right)$. Indeed, since $p>0, C_{0}, C_{1}, C_{2}$ and $C_{3}$ cannot have all the same colour. Thus $\left\{c\left(C_{0}\right), c\left(C_{1}\right), c\left(C_{2}\right), c\left(C_{3}\right)\right\}=\{1,3\}$ and then, by Proposition $4.7, m \equiv 2 \equiv 2 c=c\left(C_{0}\right)+c\left(C_{1}\right)$. This gives a contradiction, as above.

Thus, in the rest of the proof we may assume that $P$ has some red arrow. If $c\left(C_{2}\right) \neq c$ then, by Corollary 4.8, we have $c\left(C_{2}\right)=2$ and, hence, $p=0$, a contradiction. So, $c\left(C_{2}\right)=c$.

From all the above we see that $c\left(C_{0}\right)=c\left(C_{1}\right)=c\left(C_{2}\right)=c \in\{1,3\}$ and $c\left(C_{3}\right) \neq c$. Furthermore, we can also assume that there is no smallest arrow between $C_{1}$ and $C_{2}$. Otherwise, relabelling the arrows from that one on, we are in the case $c\left(C_{0}\right) \neq c\left(C_{1}\right)$, which gives a contradiction. Consequently, $n(2)+3 \leq m-(n-1)-(l-3)$.

By Proposition 4.7 we have $m \not \equiv 0, m \not \equiv c$ and $m \not \equiv 2$. Since $c \in\{1,3\}$, $3 c \not \equiv 0,3 c \not \equiv c$ and $3 c \not \equiv 2$. Therefore, $3 c \equiv m$. Then we can use Corol- 
lary 2.9 with $q=n(2)+3$ to get a periodic orbit of period $m$ with only three coloured arrows and, hence, different from $P$. This contradicts the primarity of $P$.

Let $\mathcal{C}$ be the set of all coloured arrows of a directed orbit. The above theorem states that, in the case we are interested in, $\operatorname{Card}(\mathcal{C}) \leq 3$. Hence, Theorem 4.10 is analogous to [1, Directed Theorem (d)]. The following definition gives another useful restriction about the set $\mathcal{C}$ which is also fulfilled by strongly directed primary orbits in the case $n=4$, as the next corollary shows.

Definition 4.11. A directed orbit $P$ is said to be colour compatible if each nonempty set $\mathcal{B}$ of coloured arrows satisfies

$$
\sum_{C \in \mathcal{B}} c(C) \not \equiv 0 .
$$

In particular, when $n=4$, this means that $P$ cannot have two red arrows, nor can it have green and blue arrows simultaneously. Moreover, if $P$ has three coloured arrows, then these arrows must all have the same colour, green or blue.

COROLlary 4.12. If $n=4$ and $P$ is a strongly directed primary orbit, then $P$ is colour compatible.

Proof. Assume that there is a nonempty subset $\mathcal{B}$ of the set $\mathcal{C}$ of all coloured arrows of $P$ such that $\sum_{C \in \mathcal{B}} c(C) \equiv 0$. Clearly, $\operatorname{Card}(\mathcal{B})>1$. Moreover, $\mathcal{B} \neq \mathcal{C}$ by Proposition 4.7 (a) and Lemma 2.6. Since $\operatorname{Card}(\mathcal{C}) \leq 3$ by Theorem 4.10, we have $\operatorname{Card}(\mathcal{B})=2$ and $\operatorname{Card}(\mathcal{C})=3$. That is, $P$ has three coloured arrows, $C_{0}, C_{1}$ and $C_{2}$, with $c\left(C_{0}\right)+c\left(C_{1}\right) \equiv 0$. Hence, again by Lemma 2.6, $m \equiv c\left(C_{2}\right)$, contrary to Proposition $4.7(\mathrm{~b})$.

At the beginning of this section we claimed that strongly directed orbits of maps from $\mathcal{X}_{n}$ play the same role, in the characterization of primary orbits, as directed orbits of maps from $\mathcal{Y}$. This is supported by Remark 4.2, all the results from Lemma 4.5 on and, mainly, by Theorem 4.10. It will also be supported by the fact that there exist primary directed periodic orbits of maps from $\mathcal{X}_{4}$ with an arbitrary number of coloured arrows, as the following example shows. Thus, the study of the directed primary orbits which are not strongly directed is the new feature of the characterization of primary orbits in $n$-stars with $n \geq 4$.

ExAmple 4.13. For any $p \in \mathbb{N}$, let $P$ be the directed periodic orbit of an $E P$-adjusted map $f \in \mathcal{X}_{4}$ of period $m=2 p+5$ described as follows (see Figure 4.2). The points and arrows of $P$ are labelled in the natural way, that is, $f\left(x_{i}\right)=x_{i \oplus 1}$ and $b\left(A_{i}\right)=x_{i}$ for all $i \in \mathbb{Z}_{m}$. Furthermore, for $j \in \mathbb{Z}_{4}$, $x_{j}$ is the smallest point on its branch. Since the arrows $A_{j}$ have to be black 
for $j \in \mathbb{Z}_{4}$, we see that $x_{0}<x_{4}$. Finally, we set $x_{3}<x_{5}<\ldots<x_{m-2}$ and $x_{4}<x_{6}<\ldots<x_{m-1}$.

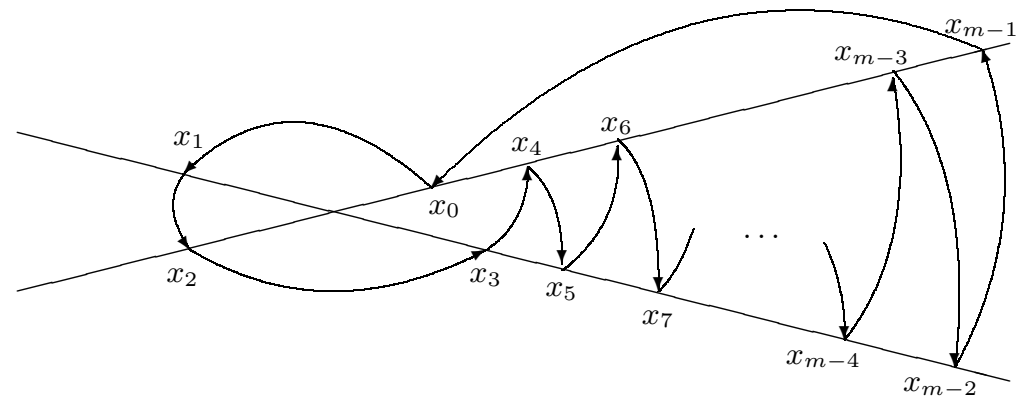

Fig. 4.2. A primary directed orbit with $p+1$ coloured arrows

Clearly this orbit has $p$ red arrows, namely, all the $A_{r}$ with $r=2 k+4$ for $k=0,1, \ldots, p-1$. It is also clear that $P$ is not strongly directed, because the arrows $A_{r-1}$ (black) and $A_{r}$ (red), for any $r$ as above, are overlapping. However, it is very easy to see that $P$ is primary. Indeed, label the basic intervals by their largest endpoints. Then in the $E P$-graph of $f$ we only have the following steps:

(i) $I_{i} \rightarrow I_{i \oplus 1}$ for each $i \in \mathbb{Z}_{m}$.

(ii) $I_{3} \rightarrow I_{0}$.

(iii) $I_{4} \rightarrow I_{1}$ and $I_{4} \rightarrow I_{3}$.

(iv) $I_{m-1} \rightarrow I_{2 k+3}$ for $k=0,1, \ldots, p$.

The steps in (i) give the fundamental loop, of length $m$. All the other shortcuts only give elementary loops of even length $(2,4, \ldots, m-3)$. Therefore, in the EP-graph of $f$, there is only one loop of length $m$, which is elementary and is associated to $P$. By [1, Propositions 1.10(b) and 1.11] we cannot get any periodic orbit of period $m$ different from $P$. Hence $P$ is primary by the First Theorem of [1].

Note that the example given in Remark 4.2 was a particular case of this one (namely, the case $p=1$ ).

This example, or maybe some particular instances of it, can be used to check that there exist other properties of strongly directed orbits which heavily rely on the Generalized Directed Rule (see, e.g., Lemma 4.6 and Proposition 4.7(b)).

From now on we only consider the case $n=4$. That is, we restrict our study to orbits of maps of $\mathcal{X}_{4}$. However we will continue using $n$ instead of 4 to unify the notation with the previous sections.

5. Box orbits for maps from $\mathcal{X}_{4}$. We shall divide the primary strongly directed orbits with more than one coloured arrow into two classes, according 
to whether they have or do not have crossing arrows in the sense of the following definition.

Definition 5.1. Two arrows $A$ and $B$ such that $b(A)<b(B)$ are said to be crossing if $e(A)>e(B)$.

Note that an extension of an orbit always has crossing arrows (see [1, Definition 3.1]).

In this section we shall see that the primary strongly directed orbits with more than one coloured arrow and with crossing arrows are precisely those defined as follows.

Definition 5.2. Let $\nu \in\{2,3\}$. We say that a periodic orbit $P$ of a map $f \in \mathcal{X}_{4}$ of period $m$ is $\nu$-box green (resp. blue) if $m$ is a multiple of $\nu$ and $P$ is an $R$-extension of a single green (resp. blue) orbit of period $m / \nu$, where $R$ is an orbit of period $\nu$ of an interval map.

REMARK 5.3. A $\nu$-box orbit has $\nu$ coloured arrows.

We shall start our study by showing that $\nu$-box orbits are primary.

Lemma 5.4. For each $\nu \in \mathbb{N}$, a $\nu$-extension of a single orbit is strongly directed.

Proof. Let $P$ be a $\nu$-extension of a single orbit. Clearly, $P$ is directed. So, we only have to see that $P$ satisfies the Generalized Directed Rule. We use the notation of $[1$, Definition 3.1] and its comments. Let $\varphi: P \rightarrow$ $\{0,1, \ldots, s-1\}$ be such that $x \in P_{\varphi(x)}$ for all $x \in P$. Then to every arrow $A=(x, f(x))$ of $P$ corresponds the arrow $\widetilde{A}=\left(y_{\varphi(x)}, \widetilde{f}\left(y_{\varphi(x)}\right)\right)$. Let $A_{0}, A_{1}, \ldots, A_{k-1}$ be overlapping arrows. Then $\widetilde{A}_{0}, \widetilde{A}_{1}, \ldots, \widetilde{A}_{k-1}$ are also overlapping because $\pi(x) \leq \pi(y)$ if $x \leq y$. Since $\widetilde{P}$ is single, $k \geq n$.

Corollary 5.5. A $\nu$-box orbit is strongly directed.

Since we are only interested in primary orbits, Lemma 5.4 together with Theorem 4.10 and Corollary 4.12 justify that, in Definition 5.2, we have considered neither the red case nor the case $\nu>3$.

Proposition 5.6. $\nu$-box orbits are primary.

Proof. Since $R$ is an orbit of period 2 or 3 in the interval, it is simple (see [1, Remark 4.18 and Definition 4.1]). Hence, by [1, Proposition 5.4], it is primary. By [1, Lemma 3.12 and Remark 3.7], it is enough to prove that if $Q$ is a single orbit of period $l$ of an $E Q$-adjusted map $h \in \mathcal{X}_{4}$, then $h$ has no periodic orbits of period $\nu l$.

Suppose that $h$ has an orbit of period $\nu l$. By [1, Proposition 1.11], we can find in the $E Q$-graph of $h$ a loop $\alpha$ of length $\nu l$ associated to that orbit. Since $Q$ is single, by Corollary 3.5 and its proof we see that all the elementary loops in the $E Q$-graph, except the fundamental one, have lengths multiple 
of $n$ and the coloured step appears only in the fundamental loop. Hence, $\nu l \equiv v l$ and $\nu l \geq v l$, where $v$ is the number of times $\alpha$ goes through the coloured arrow. Since $l \equiv c \in\{1,3\}$, it follows that $\nu \equiv v$ and $\nu \geq v$. Since $n>\nu$, we have $\nu=v$. From the equality $\nu l=v l$ it follows that $\alpha$ is a $v$-fold repetition of the fundamental loop of the $E Q$-graph of $h$. Hence $Q$ is associated to $\alpha$. By [1, Proposition 1.10] (with $n=0$ ), $\alpha$ does not give a periodic orbit of period $\nu l$, which is a contradiction.

Our next goal is to prove that the primary orbits of the class we are studying are $\nu$-box. To do this we can assume that, if $P$ is a strongly directed primary orbit of a map $f \in \mathcal{X}_{4}$ with at least two coloured arrows and with crossing arrows, then, by the First Theorem of [1], $f$ is EP-adjusted. We start with the following technical lemma.

LeMma 5.7. Let $P$ be a strongly directed primary orbit of period $m$ with $\nu>0$ coloured arrows and with crossing arrows. Then $m=\nu \cdot s$ with $\nu \in\{2,3\}, s \not \equiv 0$ and $s>n$, and there exists a partition of $P$ into subsets $P_{0}, P_{1}, \ldots, P_{s-1}$ of $\nu$ elements each such that:

(i) $\left\langle P_{i}\right\rangle \cap E=\emptyset$ for $i=0,1, \ldots, s-1$.

(ii) $P_{i}$ is in the same branch as $P_{j}$ if and only if $i \equiv j$.

(iii) $\max P_{i}<\min P_{i+n}$ for $i=0,1, \ldots, s-n-1$.

(iv) There exists $\psi:\{0,1, \ldots, s-1\} \rightarrow\{0,1, \ldots, s-1\}$ such that, if $x \in P_{i}$, then $f(x) \in P_{\psi(i)}$.

Proof. Since $P$ has crossing arrows it cannot be single by Definition 3.3. Hence $\nu \geq 2$, by Theorem 3.6. Then we can label the points and arrows of $P$ in such a way that $b\left(A_{i}\right)=x_{i}, e\left(A_{i}\right)=f\left(x_{i}\right)=x_{i \oplus 1}$ for each $i \in \mathbb{Z}_{m}$, with $A_{m-1}$ coloured and $b\left(A_{k}\right)>b\left(A_{l}\right), e\left(A_{k}\right)<e\left(A_{l}\right)$ for some $k, l \in \mathbb{Z}_{m}$ with $k \notin\{0, m-1\}$. This is clear if $A_{k}$ and $A_{l}$ are black arrows. If $A_{k}$ is coloured, then so is $A_{l}$ and we can set $l=m-1$ and clearly, $k \neq m-1$. Also $k \neq 0$ because $k=0$ gives $e\left(A_{l}\right)=b\left(A_{k}\right)>b\left(A_{l}\right)$, contradicting the Generalized Directed Rule.

Set $s m=\operatorname{sm}\left(\operatorname{br}\left(x_{m-1}\right)\right), J_{i}=\left[0, x_{i}\right]$ for $i=0,1, \ldots, m-2$ and $J_{m-1}=$ $\left[s m, x_{m-1}\right]$. Then since $x_{m-1}$ is the beginning of a coloured arrow and $s m$ is the beginning of a black arrow we see that $J_{i} \rightarrow J_{i \oplus 1}$ for each $i \in \mathbb{Z}_{m}$. Also, we have $J_{k-1} \rightarrow J_{l} \rightarrow J_{k+1}$. So we can consider the loop

$$
J_{0} \rightarrow J_{1} \rightarrow \ldots \rightarrow J_{k-1} \rightarrow J_{l} \rightarrow J_{k+1} \rightarrow \ldots \rightarrow J_{m-1} \rightarrow J_{0} .
$$

Since some of the $J_{i}$ (in fact, at least $n$ ) are basic intervals, by [1, Lemma 1.12] we can find basic intervals $I_{i} \subset J_{i}\left(i \in \mathbb{Z}_{m}\right)$ with $I_{k} \subset J_{l}$ such that $I_{i} \rightarrow I_{i \oplus 1}$ for each $i \in \mathbb{Z}_{m}$. Then, in the $E P$-graph of $f$, there is the loop

$$
\alpha=I_{0} \rightarrow I_{1} \rightarrow \ldots \rightarrow I_{m-1} \rightarrow I_{0}
$$

where each step $I_{i} \rightarrow I_{i \oplus 1}$ is of the same colour as $A_{i}$. 
If $\alpha$ is nonrepetitive, then by [6, Lemma 2.2], we get a periodic point $y \in I_{0}$ of period $m$ with $f^{i}(y) \in I_{i}$ for each $i \in \mathbb{Z}_{m}$. Set $Q=\operatorname{Orb}_{f}(y)$ and $J=\left[0, x_{l}\right]$. Note that $f^{i}(y) \in I_{i} \subset J_{i} \subset J$ for each $i \in \mathbb{Z}_{m}$ such that $x_{i} \leq x_{l}$ and $f^{k}(y) \in I_{k} \subset J_{l} \subset J$ but $x_{k} \notin J$. Therefore, $\operatorname{Card}(J \cap Q) \geq$ $\operatorname{Card}(J \cap P)+1$ because $i \neq j$ implies $f^{i}(y) \neq f^{j}(y)$ for $i, j \in \mathbb{Z}_{m}$. Then $Q \neq P$, contradicting the primarity of $P$.

Hence $\alpha$ is repetitive. Then there exists $s$ such that $m=r \cdot s$ with $r \geq 2$ and $\alpha=\beta^{r}$ with $\beta=I_{0} \rightarrow I_{1} \rightarrow \ldots \rightarrow I_{s-1} \rightarrow I_{0}$ nonrepetitive. Since $m \not \equiv 0$ by Proposition 4.7 (a), we get $s \not \equiv 0$. By Theorem 4.10, $P$ has no more than three coloured arrows, that is, $\nu \leq 3$. Hence, $I_{s-1} \rightarrow I_{0}$ is the only coloured step (of colour $c\left(A_{m-1}\right)$ ) in $\beta$ and $r=\nu$.

For each $i=0,1, \ldots, s-1$, the points $x_{i+j s}(j=0,1, \ldots, r-1)$ are all in the same branch as $I_{i}=I_{i+j s}$. Set $P_{i}=\left\{x_{i+j s}: j=0,1, \ldots, r-1\right\}$. It is clear that (i) and (iv) are satisfied with $\psi(i)=i \oplus 1$ for each $i \in \mathbb{Z}_{s}$.

Moreover, the coloured arrows of $P$ are those that begin in $P_{s-1}$ and end in $P_{0}$, that is, $A_{j s-1}$ for $j=1, \ldots, r$. From this it follows that $P_{i}$ is in the same branch as $P_{j}$ if and only if $i \equiv j$ (see Lemma 2.4). Then (ii) holds. We also see that the strings of $P$ are the sets $S_{k}=\left\{x_{i+k s}: i \in \mathbb{Z}_{s}\right\}$ for $k \in \mathbb{Z}_{\nu}$.

Consider the arrows $F_{i}$ such that $b\left(F_{i}\right)=\min P_{i}$ for $i \in \mathbb{Z}_{s}$. Since $b\left(F_{i \oplus 1}\right)=\min P_{i \oplus 1} \leq f\left(\min P_{i}\right)=e\left(F_{i}\right)$ for each $i \in \mathbb{Z}_{s}$, these arrows are overlapping. Also they are all black except $F_{s-1}$. Hence $s>n$.

Now we prove (iii). Assume that there is $j \in\{0,1, \ldots, s-n-1\}$ such that $\max P_{j}>\min P_{j+n}$. We have $\max P_{j}=x_{j+k s}$ and $\min P_{j+n}=x_{j+n+l s}$, for some $k, l \in \mathbb{Z}_{\nu}$. Moreover, since $m \not \equiv 0$ we see that $k \neq l$ in view of Proposition 2.10. Then the $\left(k \ominus_{\nu} l\right) s-n$ arrows $A_{j+n+l s}, A_{j+n+l s \oplus 1}, \ldots, A_{j+k s \ominus 1}$ are overlapping. Since $A_{j+n+l s}=F_{j+n}$ and the arrows $F_{i}$ are also overlapping we have the $m-n$ overlapping arrows

$$
A_{j+n+l s}, A_{j+n+l s \oplus 1}, \ldots, A_{j+k s \ominus 1},\left(F_{j+n}, F_{j+n \oplus 1}, \ldots, F_{j+n \ominus 1}\right)^{l \ominus k},
$$

where the notation $\left(F_{j+n}, F_{j+n \oplus 1}, \ldots, F_{j+n \ominus 1}\right)^{l \ominus k}$ means that the sequence $F_{j+n}, F_{j+n \oplus 1}, \ldots, F_{j+n \ominus 1}$ is repeated $l \ominus k$ times. Since $n$ of the arrows $F_{i}$ are the smallest arrows and $m \not \equiv 0$, we can use Lemma 2.8 to get a periodic orbit of period $m$ and different from $P$. This contradicts the primarity of $P$ by the First Theorem of [1].

Now we are ready to prove the main theorem of this section.

THEOREM 5.8. If $P$ is a strongly directed orbit with $\nu>0$ coloured arrows and with crossing arrows, then $P$ is primary if and only if $\nu>1$ and $P$ is $\nu$-box.

Proof. If $P$ is $\nu$-box, then it is primary by Proposition 5.6. Hence we only have to prove the converse. 
From Lemma 5.7 it follows immediately that $\nu>1$ and that $P$ is a $\nu$-extension of a single orbit $Q$ of period $s$. By Corollary 4.12, $Q$ is not red. If $\nu=2$ then we are done in view of [1, Remark 3.6]. So assume that $\nu=3$. With the notation of the above lemma, in view of [1, Lemmas 8.6, 8.3 and 8.1], we deduce that $f$ is monotone on all $P_{i}$ except at most one. Finally, setting $R=P_{0}$ and $g=f^{s}$, we conclude that $P$ is an $R$-extension of $Q$ (see [1, Definition 3.3]). Hence $P$ is $\nu$-box because $\operatorname{Card}(R)=\nu$.

Now we are going to show that if $P$ is strongly directed and primary, has 3 coloured arrows and its period is a multiple of 3 , then $P$ is 3 -box. This result is analogous to [1, Directed Theorem (e)], and will help us in the study of the primary strongly directed orbits with three coloured arrows.

First we prove the following lemma, which is the core of that result. In its proof, we represent by $I \stackrel{l}{\rightarrow} J$ the fact that $I f$-covers $J$ in $l$ steps, all of them black but the last, whereas $I \stackrel{l}{\rightarrow} J$ means that $I f$-covers $J$ in $l$ black steps. That is, there exist intervals $I_{i}(i=0,1, \ldots, l)$ such that $I_{0}=I, I_{l}=J, I_{i} \rightarrow I_{i+1}(i=0,1, \ldots, l-1)$, the first $l-1$ steps $I_{i} \rightarrow I_{i+1}$ $(i=0,1, \ldots, l-2)$ are black and the last step $I_{l-1} \rightarrow I_{l}$ is also black when $I \stackrel{l}{\rightarrow} J$ but it is coloured if $I \stackrel{l}{\rightarrow} J$.

Lemma 5.9. Assume that $P$ is strongly directed and primary of period a multiple of 3 with three coloured arrows. Then all three strings of $P$ have the same length.

Proof. The three coloured arrows are of the same colour, $c \in\{1,3\}$, by Corollary 4.12 (see also Definition 4.11). Then, if we denote the period of $P$ by $m$, we get $m \equiv 3 c$, by Lemma 2.6. Set $r=m / 3$ (then, of course, $r \equiv c$ ).

Suppose that the three strings of $P$ do not have the same length. Then we can label the points of $P$ in such a way that $x_{i}=f^{i}\left(x_{0}\right)$ for each $i \in \mathbb{Z}_{m}$, $x_{0}, x_{p}$ and $x_{p+q}$ are the beginnings of the three strings, $p \geq r$ and $q<r$. The lengths of the strings are, clearly, $p, q$, and $m-(p+q)$. We label the arrows in such a way that $b\left(A_{i}\right)=x_{i}$ for each $i \in \mathbb{Z}_{m}$. The coloured arrows are then $A_{p-1}, A_{p+q-1}$ and $A_{m-1}$. We are going to consider several cases and, in each of them, construct a loop of length $m$ in the $E P$-graph of $f$ which will give a periodic orbit $Q$ of $f$ different from $P$, contradicting the primarity of $P$.

First we assume that $p>r$. Since $r \equiv c$, we have $x_{p-r} \sim x_{p}$ by Lemma 2.4.

If $x_{p-r}<x_{p}$, set $L=\left[0, x_{p-r}\right]$ and $M=\left[x_{p-r}, x_{p}\right]$. Then $L f$-covers $L$ and $M$ in $r$ steps, all of them black but the last. Since $q<r$, by Lemma 2.4 we have $f^{q}\left(x_{p}\right)=x_{p+q} \nsim f^{q}\left(x_{p-r}\right)=x_{p+q-r}$. Then $0 \in f^{q}(M)$ and, hence, $0 \in f^{r}(M)$. Thus $M$ also $f$-covers $L$ and $M$ in $r$ steps, all of them black but 
the last. We consider the loop

$$
\alpha_{1}=M \stackrel{r}{\rightarrow} L \stackrel{r}{\rightarrow} L \stackrel{r}{\rightarrow} M .
$$

Suppose now that $x_{p-r}>x_{p}$. Consider the intervals $J=\left[0, x_{0}\right], K=$ $\left[0, x_{p+q}\right], L=\left[0, x_{p}\right]$ and $M=\left[x_{p}, x_{p-r}\right]$. Then $J f$-covers $L$ and $M$ in $p-r$ steps, all of them black. Since $0 \in f^{q}(M), M f$-covers $K$ in $q$ steps, all of them black but the last. Furthermore, since $0 \in f^{r}(M), M f$-covers $L$ in $r$ steps, all of them black but the last. It is also clear that $L f$-covers $K$ in $q$ steps and that $K f$-covers $J$ in $m-(p+q)$ steps, in both cases all the steps being black but the last (see Figure 5.1). We still have to consider two possibilities:

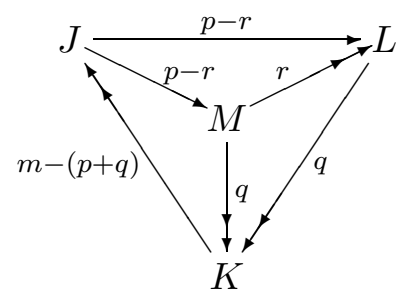

Fig. 5.1. Some $f$-coverings when $x_{p-r}>x_{p}$

CASE (a): $p+r<m$. Then since $q<r$ and $r \equiv c$ we get $x_{p+r} \sim x_{p}$ by Lemma 2.4. If $x_{p+r}>x_{p}$, then $K f$-covers $L$ in $r-q$ steps, all of them black. Then we consider the loop

$$
\alpha_{2}=M \stackrel{q}{\longrightarrow} K \stackrel{r-q}{\longrightarrow} L \stackrel{q}{\longrightarrow} K \stackrel{m-(p+q)}{\longrightarrow} J \stackrel{p-r}{\longrightarrow} M .
$$

If, on the contrary, $x_{p+r}<x_{p}$, then $x_{p+r} \in L$. Since $f^{m-(p+r)}\left(x_{p+r}\right)=x_{0}$ and $f^{m-(p+r)}(0)=0, L f$-covers $J$ in $m-(p+r)$ steps, all of them black but the last. Hence we can take the loop

$$
\alpha_{3}=M \stackrel{q}{\longrightarrow} K \stackrel{m-(p+q)}{\longrightarrow} J \stackrel{p-r}{\longrightarrow} L \stackrel{m-(p+r)}{\longrightarrow} J \stackrel{p-r}{\longrightarrow} M .
$$

CASE (b): $p+r \geq m$. That is, $p \geq 2 r$. Since $r \equiv c$, we have $x_{r-1} \sim x_{m-1}$ by Lemma 2.4. If $x_{r-1}>x_{m-1}$, then $J f$-covers $\left[0, x_{m-1}\right]$ in $r-1$ black steps and $\left[0, x_{m-1}\right] \rightarrow J$ is a coloured step. Hence we can build the loop

$$
\alpha_{4}=M \stackrel{q}{\longrightarrow} K \stackrel{m-(p+q)}{\longrightarrow} J \stackrel{r}{\longrightarrow} J \stackrel{p-r}{\longrightarrow} M .
$$

If, on the contrary, $x_{r-1}<x_{m-1}$, then $K f$-covers [0, $\left.x_{r-1}\right]$ in $m-(p+q)-1$ black steps when $p+q<m-1$, and $K \supset\left[0, x_{r-1}\right]$ if $p+q=m-1$. Since $\left[0, x_{r-1}\right] f$-covers $\left[0, x_{p-r}\right] \supset M$ in $p-2 r+1$ steps also black, $K f$-covers $M$ in $r-q$ black steps. Then we can consider the loop

$$
\alpha_{5}=M \stackrel{q}{\rightarrow} K \stackrel{m-(p+q)}{\longrightarrow} J \stackrel{p-r}{\longrightarrow} M \stackrel{q}{\rightarrow} K \stackrel{r-q}{\longrightarrow} M .
$$


If $I_{0} \rightarrow I_{1} \rightarrow \ldots \rightarrow I_{m-1} \rightarrow I_{0}$ denotes any of the above five loops (with $I_{0}=M$ ), then by using standard techniques (see, for instance, $[2$, Lemma 1.2.7]) we get a point $x \in M$ such that $f^{m}(x)=x$ and $f^{i}(x) \in I_{i}$ for each $i \in \mathbb{Z}_{m}$. Set $Q=\operatorname{Orb}_{f}(x)$. Since $0 \notin M$, we have $0 \notin Q$. Hence $Q$ has 1 or 3 coloured arrows and period $r$ or $m$, respectively. We claim that $Q$ has period $m$. This is clear in Case (b) because $p+q>2 r$, that is, $m-(p+q)<r<p+q-r$ and, hence, $Q$ cannot have only one coloured arrow. The orbits given by the loops $\alpha_{1}, \alpha_{2}$ and $\alpha_{3}$ cannot have period $r$ because, in such a case, $x=f^{r}(x)=f^{2 r}(x) \in M \cap L$, which reduces to a single point of $P$, that is, of period $m>r$. So, the claim is proved. However, the orbit $Q$ is different from $P$. Indeed, if $Q$ is given by the loop $\alpha_{1}$, then it has all three strings of the same length, $r$, unlike $P$. In the remaining cases, the strings of $Q$ have lengths $p+q-r, r$ and $m-(p+q)$ and, since $q<r<p$ and $q<p+q-r<p$, those lengths are not equal to the lengths of the strings of $P$. We then have a contradiction with the primarity of $P$.

Now assume that $p=r$. By Lemma 2.4, $x_{0} \sim x_{p} \sim x_{p+r}$ because $r \equiv c$ and $q<r$. Set $K=\left\langle x_{0}, x_{p+r}\right\rangle, L=\left\langle x_{0}, x_{p}\right\rangle, M=\left\langle x_{p}, x_{p+r}\right\rangle$ and $J=$ $\left[0, x_{p+q}\right]$. As above we will build appropriate loops to get orbits of period $m$ different from $P$, depending on the relative positions of $x_{0}, x_{p}$ and $x_{p+r}$. To do this observe first that since $f^{r}\left(x_{0}\right)=x_{p}, f^{r}\left(x_{p+r}\right)=x_{0}$ and $q<r$, we always have $K \stackrel{r}{\rightarrow} L$ and $J \stackrel{r-q}{\longrightarrow}\left[0, x_{p+r}\right]$. Furthermore, since $q<p=r \equiv c$, again from Lemma 2.4, $x_{q} \nsim x_{p+q} \nsim x_{p+r+q}$. Hence $0 \in f^{q}(L), 0 \in f^{q}(M)$ and, since $q<r, 0 \in f^{r}(L)$ and $0 \in f^{r}(M)$. This means that we also have $L \stackrel{q}{\rightarrow} J, M \stackrel{q}{\rightarrow} J, L \stackrel{r}{\rightarrow}\left[0, x_{p}\right]$ and $M \stackrel{r}{\rightarrow}\left[0, x_{0}\right]$ (see Figure 5.2).

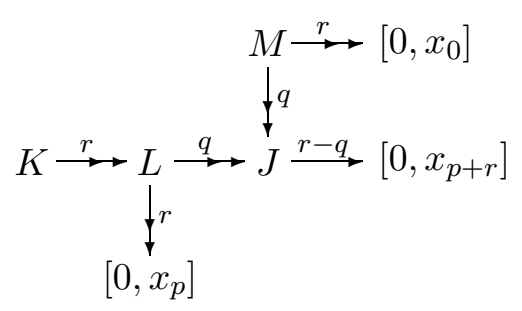

Fig. 5.2. Some $f$-coverings when $p=r$

The six possible cases are: have

(i) $x_{0}<x_{p}<x_{p+r}$. Then $L$ and $M$ are contained in $\left[0, x_{p+r}\right]$ and we

$$
L \stackrel{q}{\rightarrow} J \stackrel{r-q}{\longrightarrow} L \stackrel{q}{\rightarrow} J \stackrel{r-q}{\longrightarrow} M \stackrel{q}{\rightarrow} J \stackrel{r-q}{\longrightarrow} L .
$$

(ii) $x_{p+r}<x_{p}<x_{0}$. Then $L$ and $M$ are contained in $\left[0, x_{0}\right]$ and $M \subset$ $\left[0, x_{p}\right]$. Hence

$$
M \stackrel{r}{\rightarrow} M \stackrel{r}{\rightarrow} L \stackrel{r}{\rightarrow} M
$$


(iii) $x_{p+r}<x_{0}<x_{p}$. Then $L$ and $K$ are contained in $\left[0, x_{p}\right]$ and we have

$$
L \stackrel{r}{\rightarrow} L \stackrel{r}{\rightarrow} K \stackrel{r}{\rightarrow} L .
$$

(iv) $x_{p}<x_{0}<x_{p+r}$. Then $L \cup K \subset\left[0, x_{p+r}\right]$ and, hence,

$$
L \stackrel{q}{\rightarrow} J \stackrel{r-q}{\longrightarrow} L \stackrel{q}{\rightarrow} J \stackrel{r-q}{\longrightarrow} K \stackrel{r}{\rightarrow} L .
$$

(v) $x_{p}<x_{p+r}<x_{0}$. Then $K \cup M \subset L \subset\left[0, x_{0}\right]$ and we have

$$
M \stackrel{r}{\rightarrow} M \stackrel{r}{\rightarrow} K \stackrel{r}{\rightarrow} M .
$$

(vi) $x_{0}<x_{p+r}<x_{p}$. Now we must use the interval $\left[0, x_{0}\right]$. We have $\left[0, x_{0}\right] \cup L=\left[0, x_{p}\right],\left[0, x_{0}\right] \stackrel{r}{\rightarrow}\left[0, x_{p}\right]$ and $\left[0, x_{0}\right] \subset\left[0, x_{p+r}\right]$, hence

$$
\left[0, x_{0}\right] \stackrel{r}{\rightarrow} L \stackrel{q}{\rightarrow} J \stackrel{r-q}{\longrightarrow}\left[0, x_{0}\right] \stackrel{r}{\rightarrow}\left[0, x_{0}\right] .
$$

All these loops give an orbit $Q$ of period $m$, as we can easily see by repeating previous arguments. Namely, in all the loops there are two intervals $r$ steps apart whose intersection reduces to a point of $P$. It is also immediate that $Q \neq P$ in cases (i), (ii), (iii) and (v), since the strings of $Q$ are all of the same length, unlike those of $P$ by hypothesis. In case (iv), $Q \neq P$ because the strings of $Q$ have the same lengths as those of $P$, but in a different order: if we label the strings of $Q$ in such a way that the first is of length $r=p$, then the second has length $2 r-q>r>q$. Lastly, to show that $Q \neq P$ in case (vi), label the points $y_{i} \in Q$ in such a way that $y_{0} \in\left[0, x_{0}\right], y_{i}=f^{i}\left(y_{0}\right)$ for $i \in \mathbb{Z}_{m}$ and the beginnings of the three strings are $y_{0}, y_{r}$ and $y_{r+q}$. If $Q=P$, then $y_{r+q}=x_{p+q}$ since both are the beginning of the longest string (of length $2 r-q=m-(p+q)$ ). However, $f^{r-q}\left(y_{r+q}\right) \leq x_{0}<x_{p+r}=f^{r-q}\left(x_{p+q}\right)$. Hence, $x_{p+q} \neq y_{r+q}$; a contradiction. Then, since $Q \neq P$ in all cases we obtain a contradiction with the primarity of $P$ as before.

Now we are ready to prove the desired result.

Theorem 5.10. Let $P$ be a periodic orbit of a map $f \in \mathcal{X}_{4}$. Then $P$ is a primary strongly directed orbit of period a multiple of three having three coloured arrows if and only if $P$ is 3-box.

Proof. If $P$ is 3-box, then it has three coloured arrows by Remark 5.3, its period is a multiple of three by Definition 5.2, $P$ is strongly directed by Corollary 5.5 and it is primary by Proposition 5.6.

Now we prove the converse. The three coloured arrows are of the same colour, $c \in\{1,3\}$, by Corollary 4.12. If we denote the period of $P$ by $m$, we have $m \equiv 3 c$ by Lemma 2.6. Also, by Lemma 5.9 the three strings have the same length, $r=m / 3$. Then $r \equiv c$ and, by Lemma 2.4, the three strings begin in the same branch. Then $P$ has some crossing arrows. Indeed, assume we have labelled the points of $P$ in such a way that $x_{0}$ is the beginning of a 
string, $x_{i}=f^{i}\left(x_{0}\right)$ for $i \in \mathbb{Z}_{m}$ and $x_{0}<\min \left\{x_{r}, x_{2 r}\right\}$. Since $f^{r}\left(x_{0}\right)=x_{r}>$ $x_{0}=f^{r}\left(x_{2 r}\right)$, we can find a $t<r$ such that $x_{t}<x_{2 r+t}$ but $x_{t+1}>x_{2 r+t \oplus 1}$. Hence $P$ is 3 -box by Theorem 5.8 .

6. Conclusions. In this section we summarize the main results of the paper. The next theorem summarizes Theorems 3.2 and 3.6 about primary directed orbits of maps from $\mathcal{X}_{n}$ having at most one coloured arrow. The second one puts together Theorem 4.10 and Corollary 4.12, giving important restrictions for strongly directed orbits of maps from $\mathcal{X}_{4}$ to be primary. The last result we quote is Theorem 5.8, which characterizes those primary orbits having crossing arrows.

Theorem A. Let $P$ be a directed orbit of a map $f \in \mathcal{X}_{n}$ with $\nu \leq 1$ coloured arrows.

(a) If $P$ has only black arrows, then $P$ is primary if and only if it is twist.

(b) If $P$ has a coloured arrow $A$, then $P$ is primary if and only if it is single of colour $c(A)$.

Theorem B. Let $P$ be a strongly directed orbit of a map $f \in \mathcal{X}_{4}$. If $P$ is primary, then it has no more than three coloured arrows and it is colour compatible.

Theorem C. If $P$ is a strongly directed orbit of a map $f \in \mathcal{X}_{4}$ with $\nu>0$ coloured arrows and with crossing arrows, then $P$ is primary if and only if $\nu>1$ and $P$ is $\nu$-box.

As mentioned in the introduction, to finish the characterization of the strongly directed primary orbits of maps from $\mathcal{X}_{4}$, we are left with the case of those having $\nu>1$ coloured arrows and without crossing arrows. This will be the goal of [4].

\section{References}

[1] L. Alsedà, J. Llibre and M. Misiurewicz, Periodic orbits of maps of $Y$, Trans. Amer. Math. Soc. 313 (1989), 475-538.

[2] - - - 一, Combinatorial Dynamics and Entropy in Dimension One, 2nd ed., Adv. Ser. Nonlinear Dynamics 5, World Sci., River Edge, NJ, 2000.

[3] L. Alsedà, J. Llibre and R. Serra, Minimal periodic orbits for continuous maps of the interval, Trans. Amer. Math. Soc. 286 (1984), 595-627.

[4] L. Alsedà and J. M. Moreno, On the primary orbits of star maps (second part: spiral orbits), Appl. Math. (Warsaw), to appear.

[5] S. Baldwin, Generalizations of a theorem of Šarkovski on orbits of continuous realvalued functions, Discrete Math. 67 (1987), 111-122. 
[6] S. Baldwin, An extension of Śarkovskiù's Theorem to the n-od, Ergodic Theory Dynam. Systems 11 (1991), 249-271.

[7] L. Block, Simple periodic orbits of mappings of the interval, Trans. Amer. Math. Soc. 254 (1979), 391-398.

[8] W. A. Coppel, Šarkovskii-minimal orbits, Math. Proc. Cambridge Philos. Soc. 93 (1983), 397-408.

[9] C.-W. Ho, On the structure of the minimum orbits of periodic points for maps of the real line, preprint, Southern Illinois Univ., Edwardsville, IL, 1984.

[10] L. Snoha, Minimal periodic orbits of mappings of an interval, Zeszyty Nauk. WSP w Bydgoszczy, Problemy Matemat. 1985, z. 7, 146-149.

[11] - Characterization of potentially minimal periodic orbits of continuous mappings of an interval, Acta Math. Univ. Comenian. 52-53 (1987), 111-124.

[12] P. Stefan, A theorem of S̆arkovskii on the existence of periodic orbits of continuous endomorphisms of the real line, Comm. Math. Phys. 54 (1977), 237-248.

Departament de Matemàtiques

Facultat de Ciències

Universitat Autònoma de Barcelona

08193 Cerdanyola del Vallès, Barcelona, Spain

E-mail: alseda@mat.uab.es
Departament de Matemàtica Aplicada II

E.T.S.E.I.T.

Universitat Politècnica de Catalunya 08222 Terrassa, Barcelona, Spain

E-mail: moreno@ma2.upc.es 